\title{
TREATMENT OF PLANTAR TROPHIC ULCERS WITH "NOVOLEP" IN A RURAL LEPROSY CENTRE
}

\author{
by V. EKambaram, M.B., B.S.
}

and C. S. Gangadhar Sharma, M.B., B.S.

Senior Medical Officer in Charge and Medical Officer respectively of the Government Leprosy Treatment and Study Centre, Tirukoilur, $S$. India

\section{Introduction}

Though the sulphones have revolutionised the treatment of leprosy there is the still unsolved problem of the treatment of trophic ulcers. The modern consensus of opinion about the causation of these is that anaesthesia is not directly responsible for the ulcers but is only a predisposing and perpetuating factor. The patient who does not take care of his anaesthetic feet sustains repeated minor injuries which lead to trophic ulcers. The occupation of patients in a rural area is mainly agriculture, which is hazardous in that there are many unavoidable exposures to injury of the limbs and feet, for they are poor and have to work barefooted, and sustain pricks from thorns, and bruises and cuts from stones and the jagged cut ends of plants. There is a great lack of machine aid to agriculture in India and all the work has to be done by man power. Even those who can afford footwear do not use it. So in this rural leprosy centre we have patients who are not intelligent enough to take care of their feet but are subject to the risk of many injuries. We have no X-ray, so found difficulty in gaining knowledge of the condition of the bones underlying the ulcers, but found probing useful and studied well the clinical appearance and chronicity, a course which is open to all field workers.

We found 124 cases of trophic ulcers among the patients in this area and had to hospitalize 89 of them during the year 1958. During the last quarter of 1957 we began trial of a product called Novolep, which is a bye-product of the manufacture of DDS and is supplied by the Bengal Chemical and Pharmaceutical Works, Calcutta. DHARMENDRA in 1955 had reported encouraging results with it in Leprosy in India of July that year. He used it as an application and found it to be more satisfactory than other dressings in common use. Complete and permanent healing could not be expected if dead bone was involved, for which surgical measures are also needed, and steps must also be taken to improve the circulation of the part, and to protect it against constant pressure and repeated injuries.

Novolep is a bye-product of the manufacture of DDS (Novophone) by the process adopted by Dr. H. C. Biswas of the Bengal Chemical and Pharmaceutical Works, and was supplied to us as 
a liquid in one gallon tins. It contains a small amount of DDS and a large percentage of alcohol. With a Specific Gravity of 1.01 to 1.02 the preparation has a total solid content of $40 \%$, with $60 \%$ rectified spirit. In the solid content there is mainly DDS, with traces of 4-nitro-4-amino-diphenylsulphone and of iron. The former differs from DDS in solubility, melting point, microscopic appearance, and chemical composition.

\section{Selection of Cases}

All the patients had had the routine ulcer treatment for quite a while without improvement, and oral sulphone therapy was kept up during the trial. We chose cases who had ulcers for at least six months without benefit from the routine dressings. We took the first batch as in-patients during the trial, for closer observation, rest, and better food, but a second later batch we formed of out-patients so as to study results when they were pursuing their normal occupations. In September, 1957, the first batch began the trial: there were 11 cases, of whom four were lepromatous and seven non-lepromatous. There were six cases with a single ulcer and five with multiple ulcers. The duration of the ulcers was six months to one year in four cases, one year in three cases, two years in one case, and three years in three cases. There was bone involvement in three cases. The ulcer sites were: metatarsal heads in seven cases, lateral border of the foot in one case, heel in two cases, and there was one other site. Treatment by the new application was given for $2 \frac{1}{2}$ to 10 months.

\section{Method of Treatment}

In the event of any covering of slough or undermining of the edges of the ulcers on admission, the ulcers were dressed with magnesium sulphate-glycerine for a few days and the edges trimmed: the dressing was continued for a few days until the ulcers were cleared of slough. If there was any rise of temperature or local oedema, antibiotics were also given for a few days. After that the ulcers were dressed daily with gauze soaked in Novolep and a light bandage kept on the gauze. The patient was told to avoid unnecessary walking, and for going to the latrine at the rear of the hospital, a piece of rubber sheeting was provided to be wound round the foot to protect the bandage or ulcer from mud and earth.

The second batch containing out-patients was formed towards the end of 1958, because in the first batch hospitalization brought in rest and other protective factors, and the estimation of the value of the treatment to the ambulatory was of such great practical importance.

Out of 17 out-patients 11 carried through with the treatment, and of these four were lepromatous and seven non-lepromatous. 


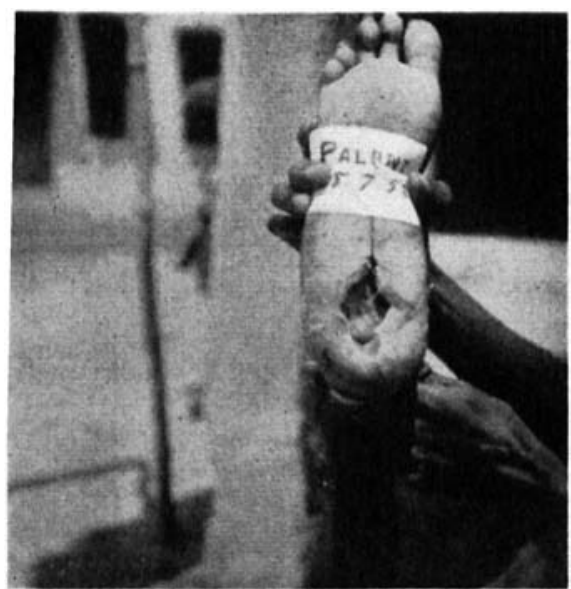

$5-7-58$

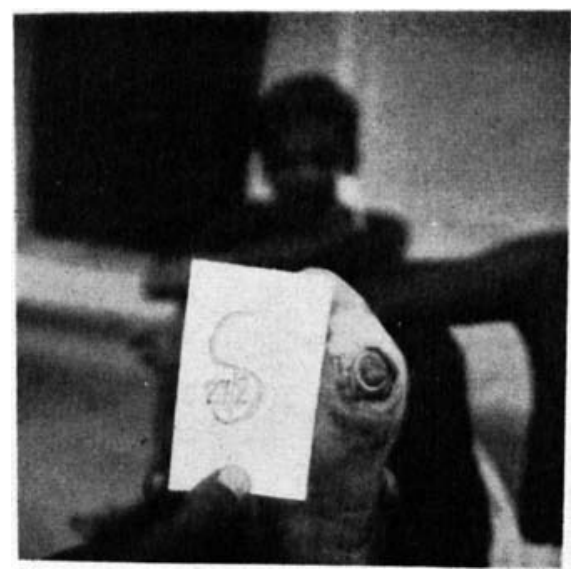

$27-2-58$

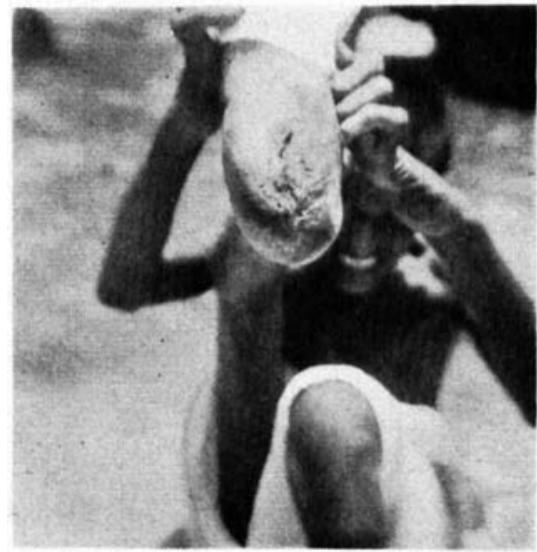

May 1959, Almost healed

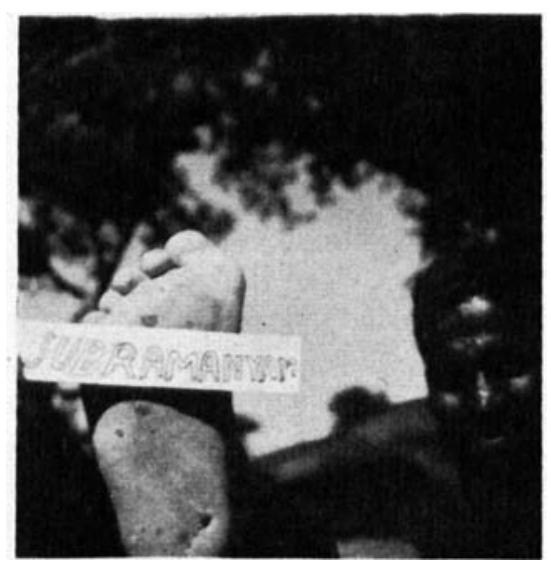

4-4-58, Healed well 


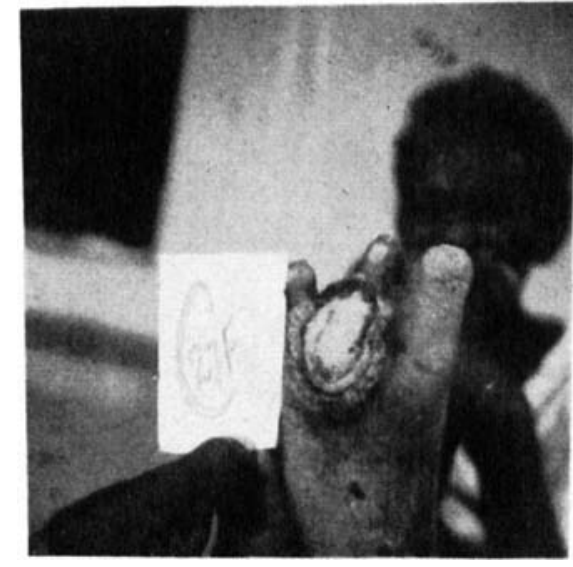

$27-12-58$ Right foot

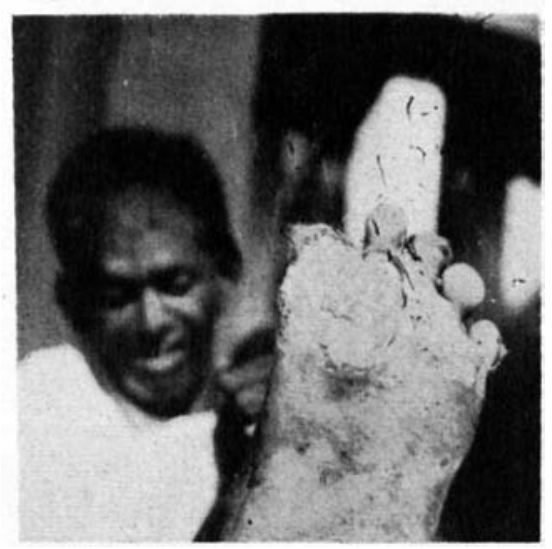

Same patient 27-12-58 Left foot

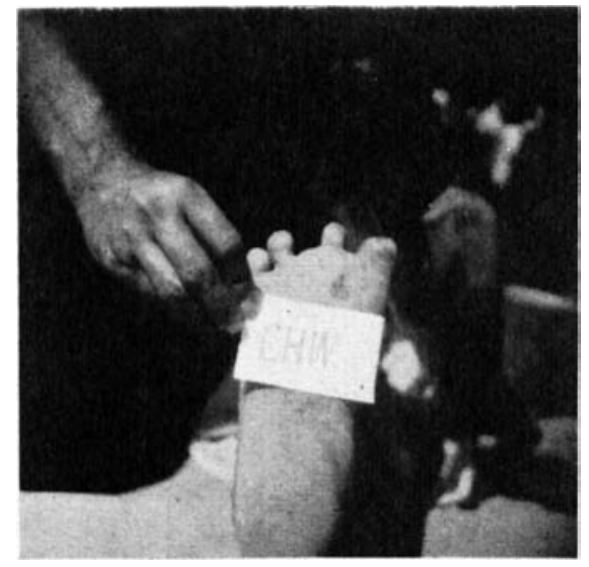

4-4-59, Healed well, right foot

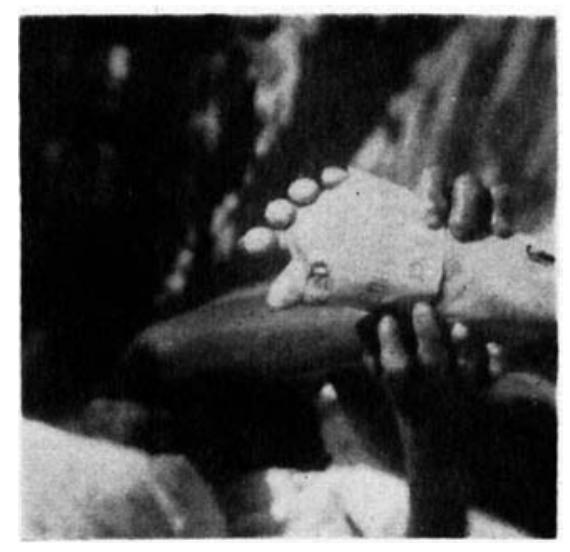

13-6-59, Not healed completely even after one year six months dressing with Eusol etc. 
They had ulcers of two years' duration in two cases, of four years in one, and five years in one, of six years in one, and of one to two years in one, of six months to one year in five. There were single ulcers in seven cases and multiple ulcers in four. There was bony involvement in three cases only. The ulcers lay over the metatarsal heads in 10 and on the heel in one. Each patient was receiving outpatient oral sulphone therapy and first was asked to come to the headquarters clinic to receive the same preliminary treatment as in the first batch. After the ulcer had thus been cleaned up each patient was given enough Novolep in a bottle to last for a week and dressing materials of gauze, cotton, and three or four rolls of bandage, and Eusol with which to clean the edges of the ulcer. He was taught how to use this and how to apply the Novolep with a piece of gauze soaked in the preparation, and to apply a light bandage, and advised to tie a piece of rubber sheeting for walking or doing any work which might soil the bandage, and advised to avoid injuries to the feet.

\section{Results}

The results among the in-patient group were much better than in the out-patient, probably because of the important additional factor of rest. Of the in-patients, a mong 11 who continued treat ment, only one had no healing and in that there was clinical evidence of the involvement of bones. Of the other 10 cases, six healed completely and four partially. There was one case of bone involvement among the six who healed completely. This patient had an ulcer at the base of the big toe and of the fifth toe, and in the former ulcer the bone could be felt on probing. In these six successful cases treatment lasted $2 !$ to 10 months and the duration before treatment was less than one year except for one case of three years' duration. Among these six cases one patient had an ulcer of almost equal dimensions on each foot; the right side was treated with Novolep and the left with routine dressings of Eusol. That treated with Novolep healed in four months whereas that treated in the routine manner took over one year. Again, of the six well healed cases, three relapsed at the same ulcer site within two months of their discharge. Among the four cases partly healed, three became worse after discharge from hospital. Clinically detectable bone involvement occurred in one of the four partly healed cases: the period of treatment of these cases ranged from four to 10 months. Many of the ulcers were of three years' duration before treatment. In general, ulcers where bone involvement was clinically detectable responded less well than those without it, and ulcers of longer duration and hence indolent in nature took a longer time to heal.

The occurrence of relapses must mean that the healing was of only temporary nature, or else the patients after discharge had 
broken the rules of protection and prevention. It was noted that healing set in quickly after Novolep was used. The edges of the wound grew up quickly from the sides without parallel growth from below, with the result that the apparently healed ulcers broke down into their original state as soon as the patient began walking. Only ulcers without any bone involvement healed with lasting effects.

In the out-patient group there were definitely poorer results. Of the 11 cases who continued the treatment, three healed completely, five only partially, and three showed no healing at all. Among the three cases of complete healing, two patients had two ulcers each, but only one ulcer healed in each patient, though bone involvement was not detectable. The duration of treatment in these cases ranged from one and a half to three months. Superficial ulcers of shorter duration healed more quickly and completely than the others. Among the 5 cases which healed only partly up to June, 1959, there was only one case of bone involvement, which healed partly after seven months' treatment. Also one case with an ulcer of six years' duration without bone involvement healed only partly despite treatment for four months. This latter case was changed to a different method of treatment. The remaining three cases did improve definitely but then became stationary after four to nine months. The three cases with no response to Novolep showed no healing nor decrease of the discharge from the ulcers, but 2 of these cases had definite involvement of bone and improved after removal of the necrosed bone; but one case with an ulcer on the planter aspect of each toe is still not healed, though without involvement of bone. Of the three completely healed cases, one relapsed in three months with an ulcer at the same spot.

In general, therefore, as soon as the dressing with Novolep is started there is an apparent improvement in the ulcer. The ulcer discharge lessens, and the ulcer looks better and there appears to be an attempt at quicker healing. The ulcer edges grow quickly to close the wound but basal growth lags behind. Though encouraging, this apparent healing is not maintained. Even among completely healed cases, half of them relapsed later from repetition of the same causes as operated in the first place. Results in cases with bone involvement are poor, likewise generally in out-patients. The factors of rest and avoidance of trauma are all-important. The rate of healing under Novolep is definitely quicker than with other applications, but there was one case of chronic ulcer of six years' duration which has not improved at all, even in the absence of clinically detectable involvement of bone. On the other hand, one of the in-patient cases did have bone involvement but healed completely.

\section{Summary}

Novolep treatment of trophic ulcers of the feet was tried in two 

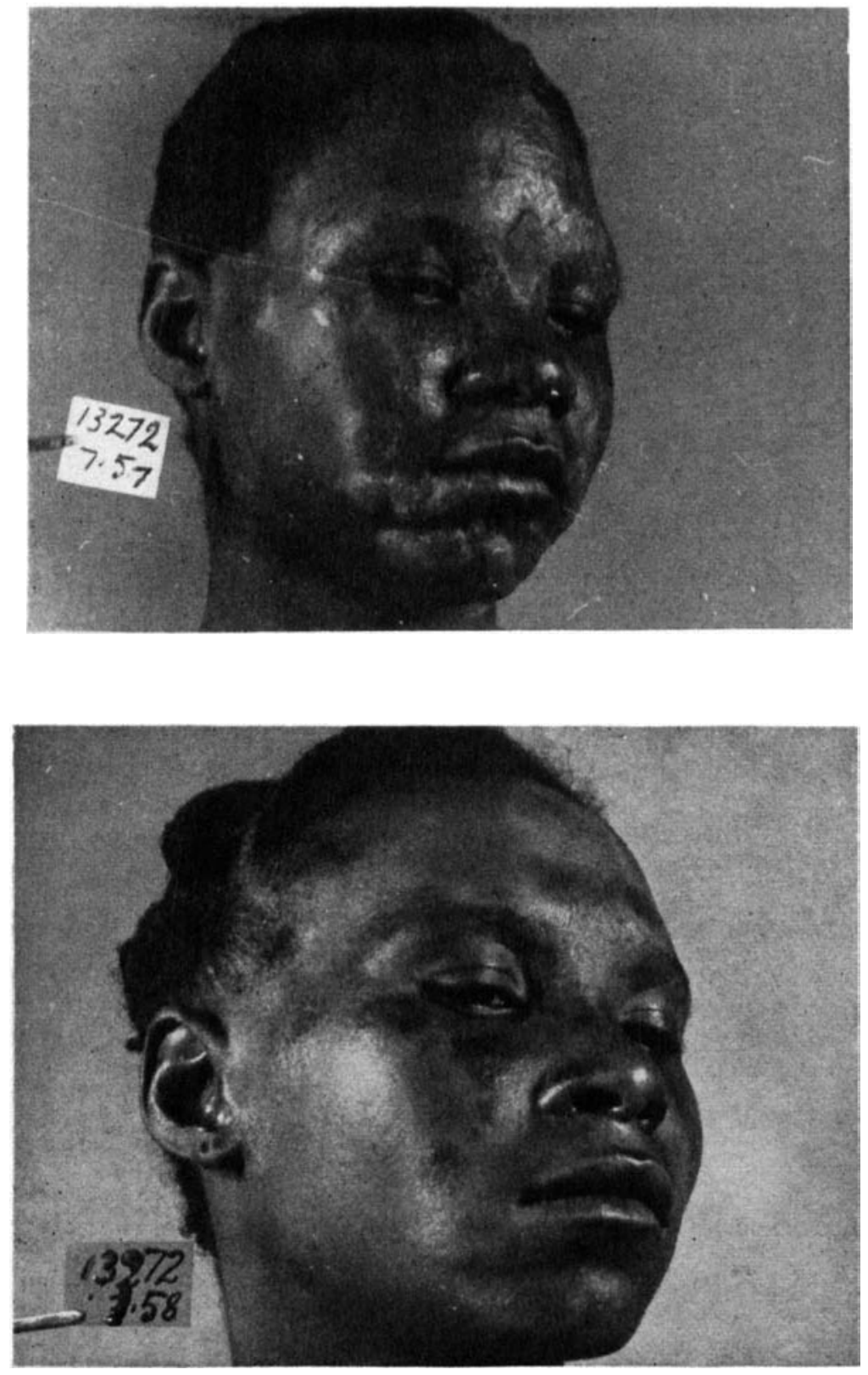

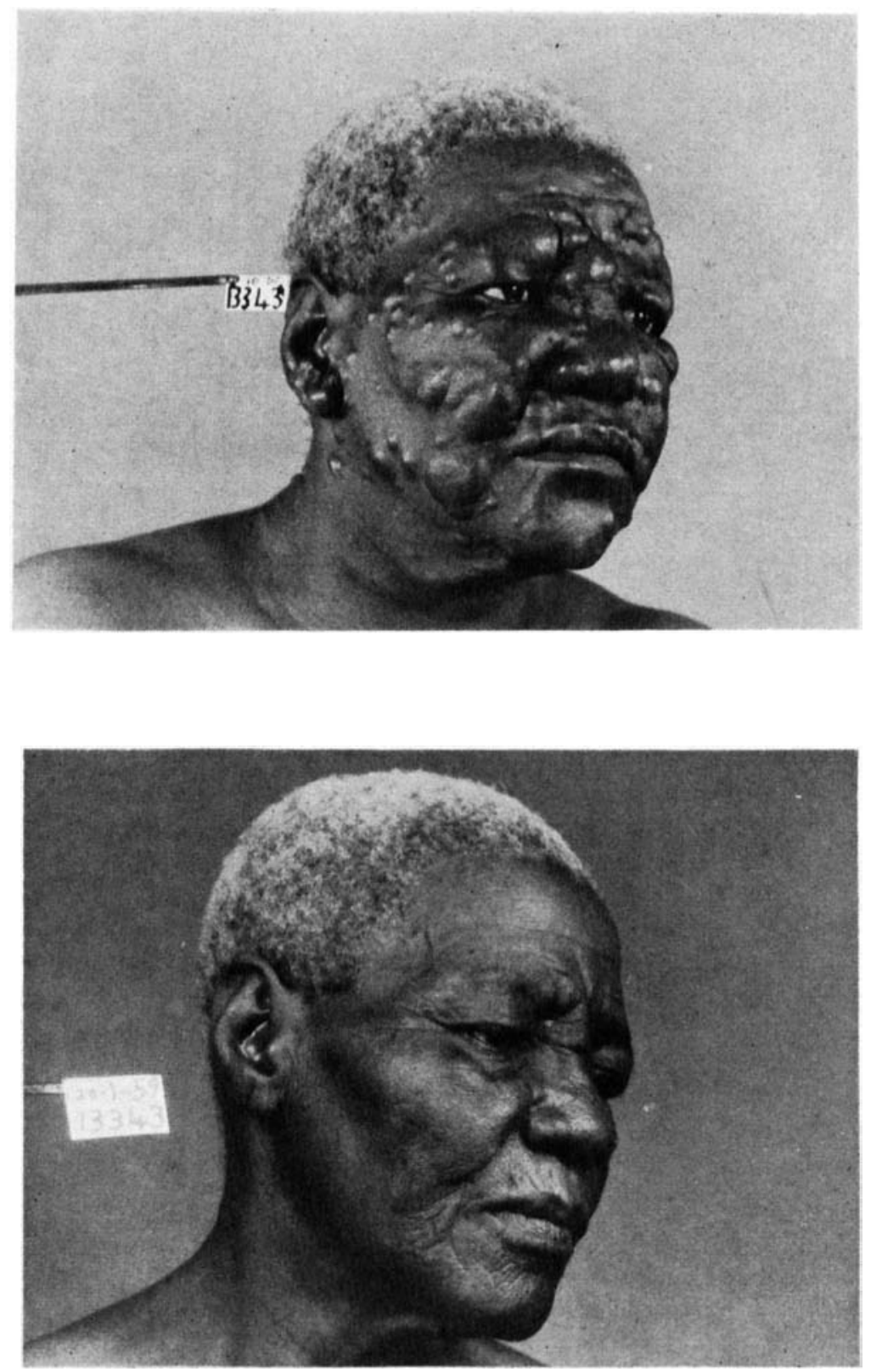
groups of patients. one in-patient and the other out-patient. There were better results in the first, though there were relapses after discharge from hospital. The out-patient group gave poorer results, probably because they were exposed to repeated trauma. Healing with Novolep is quicker than with other medicaments, provided there is no involvement of bones. A few ulcers with extensive damage to soft tissues and with gross infection have responded to Novolep and they probably would not have healed with other applications, except with surgical intervention. The care of the feet and protection from trauma remain as essential as with all other treatments. Further field trials are necessary before any definite conclusion about Novolep can be given.

\section{Acknowledgement}

Our grateful thanks are due to Dr. K. Ramanujan, M.B., B.S., Medical Officer, Silver Jubilee Children's Clinic, Saidapet, Madras, for his valuable suggestions, and guidance in the preparation of this article; also to Dr. H. G. Biswas, Bengal Chemical and Pharmaceutical Works, Ltd., Calcutta, for kindly supplying us with one gallon of Novolep for clinical trial, and to our Hospital Assistants for their help in this work, and also to the patients for their active co-operation.

\section{Note by the Editor}

Two tables of clinical notes and some of the illustrations have had to be left out, for reasons of space. 